\title{
LA EDUCACIÓN A DISTANCIA: \\ UNA INVITACIÓN A LA CONSTRUCCIÓN CONJUNTA
}

DISTANCE EDUCATION: AN INVITATION

TO A MORE COMPREHENSIVE APPROACH

Marta Susana Brovelli 1
Resumen Con este artículo se pretende continuar con el debate y las reflexiones acerca de la Educación a Distancia (EaD). Para ello se propone: en primer lugar, algunas consideraciones generales que tienen que ver con la Educación a Distancia y el uso de las Nuevas Tecnologías de la Información y de la Comunicación (NTIC) y su desarrollo en la sociedad actual. En segundo lugar, y pensado muy especialmente en la formación de formadores, se pasa a plantear la necesidad de construir una 'didáctica crítica', que rompa con los modelos de tipo tecnicista que hasta hoy han sido preponderantes en la EaD. De ese modo, se intentan recuperar o bien abordar las dimensiones políticas y éticas de la didáctica y el desarrollo de sujetos y profesionales críticos.

Palabras clave educación a distancia; democratización de la enseñanza; didáctica crítica; profesionales críticos.
Abstract This article intends to give continuity to the debate on - and the reflections about Distance Education (EaD). With this purpose in mind, we make, in the first place, some general considerations about this type of education and the use and development of the New Information and Communication Technologies (NTIC) in present society. Secondly, and mostly in connection with teachers' training, we suggest that there is a need for a 'critical didacticism', able to break away from the technicist models that, to date, have predominated in EaD. For this purpose, we try to recover or to approach the political and ethical dimensions of teaching and those that encourage the development of critical subjects and professionals.

Key words distance education; democratization of teaching; critical didacticism; critical professionals. 


\section{Introducción}

En primer lugar, quiero agradecer sinceramente la invitación de la revista Trabalho, Educação e Saúde a publicar, a dialogar, a debatir e intercambiar experiencias entre aquellos que estamos en el campo educativo y formativo. Debo aclarar que mi participación está dada desde mi condición de profesional de las Ciencias de la Educación, y más especialmente del campo curricular y didáctico.

El problema de la Educación a Distancia (EaD) y el uso de las nuevas tecnologías de la comunicación han suscitado mi interés y el de muchos profesionales, en razón de su avance continuado y en incremento, por lo que considero que hoy constituye una nueva modalidad de enseñanza y de formación, especialmente en el posgrado, que ha llegado para quedarse. De allí el valor e importancia que tienen las reflexiones teóricas y prácticas que puedan realizarse en torno a ella y a sus características básicas, con el propósito de incrementar saberes y prácticas de lo que podríamos llamar 'un nuevo campo educativo'.

Es indudable que en lo referido a la formación permanente de profesionales en el área de la salud y específicamente de la enfermería, la modalidad a distancia parece haber adquirido una importancia relevante, sobre todo en países como Brasil y Argentina, por la necesidad de brindar profesionales capacitados para llegar a diferentes y lejanas zonas y localidades, o bien para mejorar los servicios en centros de salud de las ciudades. Para satisfacer esta necesidad sería prácticamente imposible hacerlo mediante los procedimientos y medios tradicionales. Es precisamente aquí donde la EaD ha mostrado mayor efectividad, rompiendo las barreras de tiempo y espacio, al ofrecer métodos, técnicas y recursos que hacen más efectivo y flexible el proceso enseñanza y aprendizaje, mediante el uso de diversas tecnologías de la comunicación.

Una experiencia semejante a la vuestra es la que está realizando, desde hace varios años, la Escuela de Enfermería de la Facultad de Ciencias Médicas de la Universidad Nacional de Rosario, unidad académica con la que he tenido relaciones de asesoramiento. Esta escuela ofrece la Licenciatura en Enfermería de forma presencial y a distancia. Cuenta con varias subsedes en el país, al punto que en la actualidad la actividad a distancia está ocupando un lugar preponderante en sus tareas docentes.

Es pertinente reconocer que, más allá de los motivos de tipo pedagógico, la potenciación de la modalidad a distancia puede tener repercusiones sociales importantes. Por un lado, abre una vía a la democratización de la enseñanza, ya que ofrece más facilidades de acceso a la formación, y, por otro, facilita la concreción de proyectos político-sociales que tengan que ver con el desarrollo de derechos humanos, formación de ciudadanos críticos, 
capaces de cuidarse y demandar en función de sus necesidades como sujetos sociales.

Este rápido desarrollo de la modalidad a distancia, así como lo que podríamos llamar el 'éxito' de esta nueva oferta educativa - que puede ser considerada como una innovación - y la consecuente ocupación de los profesores en tareas para las que no fueron específicamente preparados, y que ocupan gran parte de su tiempo laboral, es precisamente lo que otorga fundamentos suficientes para preocuparnos por esta modalidad de enseñanza y de aprendizaje. Con una finalidad, la de comprometernos en una tarea con evidentes efectos sociales, que requiere de reflexiones y acciones que aseguren las mejores posibilidades de una educación y formación de calidad.

Atendiendo a lo expresado anteriormente, me permito coincidir ampliamente con el título del artículo que me enviara la revista: Educación a distancia y formación en salud: ni tanto ni tan poco, y es por lo que intentaré desarrollar algunas cuestiones que tienen que ver, fundamentalmente, con pensar una didáctica de la EaD que responda a una perspectiva 'crítica' del campo, que reconozca la dimensión política y ética de la enseñanza, que es su objeto de estudio. Objeto que requiere siempre de la necesaria contextualización y del análisis de las intercomunicaciones que se producen en cada situación particular en la que tiene lugar.

\section{¿Por qué ocuparnos de las NTIC y de la EaD que las incluye?}

Partimos de considerar a las Nuevas Tecnologías de la Información y de la Comunicación (NTIC) como una producción social que está provocando, y seguirá haciéndolo, cambios sociales complejos y profundos. Hasta tal punto que hoy se habla de la 'revolución tecnológica', así como antes se ha considerado a la revolución neolítica y la revolución industrial. Hablamos de revoluciones atentos a las transformaciones sociales que produjeron.

Tal como nos enseña la Historia, estas revoluciones han sido inevitables, aunque han tenido diferentes ritmos y tiempos. La revolución tecnológica, aún desde la provisionalidad del presente, observamos que tiene características trepidantes, cuyos efectos e impactos se expanden a la velocidad de las ondas de una explosión. De allí la importancia de los estudios anticipatorios y de las reflexiones acerca de los problemas sociales que pueden ir suscitándose.

La EaD, que cuenta con antecedentes dispersos antiguos, pero que como modalidad sistemática de educación tiene corta historia, también registra un avance vertiginoso con posibles efectos aún no bien estudiados, especialmente a partir de la inclusión de las nuevas tecnologías como el uso de internet y el trabajo con redes de comunicación. 
Dejando claro entonces la inevitabilidad de estos cambios tecnológicos, sociales y el avance que ha tenido y tendrá la EaD, cabe preguntarnos: ¿Qué es lo que puede evitarse o reorientarse?

\section{Desde el punto de vista social}

En primer lugar, por lo que puede observarse y anticiparse, es que esta nueva sociedad tecnologizada no viene a ofrecer soluciones a los grandes problemas sociales de exclusión y de concentración de la riqueza y del poder económico financiero globalizado y hegemónicamente manejado, que implica también el control de las informaciones y de las comunicaciones, sino que, seguramente, los está profundizando.

De este modo, el mito de una mayor democratización social se cae ante datos como los siguientes: de las 300 empresas de información y comunicación más importantes del mundo, 144 son norteamericanas, 80 de la Unión Europea, 49 japonesas; de las 75 agencias de prensa más importantes, 39 son americanas, 19 europeas, 7 japonesas; de las 158 empresas más importantes que producen material para la comunicación, 755 son norteamericanas, 36 europeas y 33 japonesas (Aparici, 2002, p. 8).

Por otra parte, si consideramos el porcentaje de personas interconectadas, es decir con acceso a internet, podemos tener otra sorpresa ya que datos de 1998 nos señalan que sólo el 2\% de los habitantes del mundo gozan de este beneficio, según datos de la misma fuente.

Sin embargo, teniendo en cuenta afirmaciones de Castells, la nueva economía es "información-al" (1994, p. 16) porque la productividad y la competitividad de las unidades o agentes de la economía (sean empresas, regiones o naciones) dependen fundamentalmente de su capacidad para generar, procesar y aplicar con eficacia la información basada en el conocimiento. Es también global porque la producción, el consumo y la circulación y componentes están organizados a escala global. Se puede afirmar que existe una singular conexión entre el conocimiento y la información. Las nuevas tecnologías de la información, es decir la internet y los satélites, son los medios que permiten el fenómeno de la globalización.

Está claro que no es posible pensar que la tecnología aplicada a los distintos ordenes de la vida social sea una panacea para la solución de los problemas, ni para una vida mejor. Esto no quiere decir que no se reconozcan los beneficios que la NTIC ha traído a la vida cotidiana de muchos ciudadanos. Permitiendo una rápida e inmediata comunicación de tipo planetario, accediendo a un volumen de información que antes nos hubiera ocupado mucho más tiempo o nos hubiera resultado imposible. Lo que pretendo plantear es que ante esta nueva realidad, que muchos han naturaliza- 
do, es conveniente colocarnos en una posición de alejamiento de la tecnofilia y de la tecnofobia, para poder pensar críticamente en los usos sociales y educativos de las NTIC.

El otro gran desafío de la EaD es el de ofrecer realmente oportunidades de formación y educación de calidad en todas las ofertas que se realicen. Ésta no es una tarea sencilla, en tanto requiere de capacidades y de competencias por parte de los profesores, diferentes a las que exige la educación presencial, así como también modos diversos de trabajo de los estudiantes. No obstante, no hay otro camino que la búsqueda y el logro de la mejor calidad. A ello deben apuntar todos nuestros esfuerzos. Ésta será también la

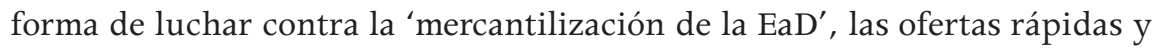
superficiales que atienden demandas artificiales y superfluas y que, por parte, desde los posibles estudiantes pueden ser percibidas como capacitaciones laborales rápidas y fáciles.

\section{Desde el punto de vista de la educación y específicamente de la EaD}

La EaD, vista desde tecnologías utilizadas, ha pasado por cuatro grandes etapas. Primera etapa: caracterizada por el dominio del material impreso, textos y manuales, por correspondencia e intercambio de documento. Segunda etapa: denominada analógica y caracterizada por la utilización de televisión, vídeos y programas radiofónicos. Tercera etapa: se incorpora la informática a los procesos de producción tecnológica de materiales. Cuarta etapa: se denomina digital, en la que se integran los diferentes medios tecnológicos a través de redes como internet $\mathrm{u}$ otros canales de distribución digital.

Actualmente, en la EaD las tecnologías más utilizadas constituyen una mezclan de los medios de las diferentes etapas, con el posible predomino de alguno de ellos.

La EaD analizada desde el punto de vista social está ligada al principio de democratización de enseñanza en tanto se planteó como una alternativa válida para llegar a aquellos sectores de la población que no podían acceder a estudios presenciales de manera sistemática y continuada. Desde ese lugar ha cumplido, y sigue haciéndolo, con este objetivo, si bien los estudios de investigación realizados demuestran que la mayor población de estudiantes proviene de zonas urbanas y son personas que trabajan, amas de casa o adultos que deciden realizar otros estudios superiores. Este aspecto estaría mostrando la importancia de esta modalidad de enseñanza en el desarrollo de la educación a distancia, que es otro de los reclamos de la sociedad actual y lo será más aún en el futuro. En este sentido se piensa que los sujetos deberán cambiar varias veces de actividad laboral durante el transcurso de su vida. 
Sin embargo, cabe señalar que en el caso de la EaD no se ha producido aún un debate profundo acerca de sus implicancias sociales, políticas e ideológicas, ya que la mayoría de los estudios realizados hasta el momento han tenido que ver con su contraste con la enseñanza presencial o con la defensa del modelo que las distintas universidades ofrecen.

La EaD, analizada desde el punto de vista educativo, tiene varios desafíos que afrontar, haciéndose cargo de las ventajas y desventajas que presenta frente a la educación presencial que conocemos.

En primer lugar, tiene que comprometerse, como todo tipo de educación superior y de posgrado, con la formación y desarrollo de una ciudadanía local y global y luchar por conseguir una sociedad productiva que también sea más justa y cohesiva.

En segundo lugar, debe tratar de lograr legitimación social y académica en cuanto a la calidad de los estudios que ofrece y certifica. Para ello debió luchar contra ciertos prejuicios que tuvieron que ver con antecedentes asistemáticos y poco serios en sus orígenes, como algunos cursos 'por correspondencia'. Este desafío lo ha superado ampliamente, especialmente, en algunos países que han podido demostrar el alto nivel académico de sus licenciados universitarios y de estudios de posgrado. Esto requerirá de investigaciones y sistemas de evaluación que atiendan a su especificidad pero que permitan la comparación con licenciados de las modalidades tradicionales.

En tercer lugar, tiene que sostener el principio de democratización de la educación. Lo que supone al menos dos cuestiones de distinto orden: una, referida al acceso de grupos de personas que encuentran dificultades para hacerlo de modo presencial, logrando, al mismo tiempo mejor retención de la matrícula que la enseñanza presencial. La segunda cuestión está referida a su capacidad para construir un modelo pedagógico más abierto y flexible, de modo que atienda a las posibilidades individuales de los sujetos, a crear un contexto de aprendizaje que permita el desarrollo de la autonomía y las posibilidades creativas, rompiendo con el rígido cerco del aula tradicional.

Los desafíos anteriores implican a su vez:

1) La formación de profesores con el mejor nivel académico posible y con las competencias específicas que requiere esta modalidad, tales como las capacidades para producir materiales con el mayor nivel científico y de comunicabilidad, así como la capacidad para trabajar cooperativamente con equipos de tutores y de técnicos de diseño de materiales y de manejo de los medios que se utilicen. También se hará necesario que la formación docente atienda a la comprensión de los alcances y limitaciones de la sociedad de la información en lo que se refiere a la defensa de los derechos sobre la circulación de la información y difusión de datos.

2) Construir modelos didácticos que no sean la reproducción de los modelos autoritarios y de racionalidad tecnicista que han predominado, y aún 
lo siguen haciendo, en la enseñanza presencial. Me refiero a metodologías de carácter unidireccional en cuanto a la comunicación, con planteos estructurados de enseñanza y de aprendizaje en los que los estudiantes tienen muy pocas posibilidades de interactuar de manera reflexiva y crítica. Para ello habrá que poder diseñar metodologías de enseñanza y de aprendizaje que rompan con la uniformidad de los contenidos, permitiendo el acceso a diferentes fuentes de conocimiento y a la mayor diversidad posible con la operacionalidad de los mismos y su articulación con situaciones de la teoría y de la práctica.

El recorrido por la corta pero fructífera historia de esta modalidad de enseñanza y los fundamentos de carácter social y políticos sobre los que se sustenta, justifican plenamente su amplio y continuo desarrollo. Sin embargo, hay que reconocer que el modelo de enseñanza predominante se encuentra impregnado de características tecnicistas, propias del modelo de enseñanza por objetivos. Allí el control y la eficiencia - medida por la relación entre lo previsto y lo logrado - se convierten en una obsesión que no da lugar a lo imprevisto, a la multicausalidad, a la subjetividad y otras notas que se encuentran hoy suficientemente validadas, como propias de los procesos de enseñanza y de aprendizaje. Esto es claro indicio de que la epistemología hegemónica en la EaD y en la Tecnología Educativa ha sido de carácter técnico-racional, propia del positivismo, dando la espalda u obviando las perspectivas sociocríticas y culturales en el campo educativo y didáctico.

Lo anteriormente dicho no niega los esfuerzos y experiencias de especialistas en EaD por producir propuestas didácticas que se sustenten en principios de una pedagogía constructivista, que atienda a los procesos subjetivos en el aprendizaje. Sin embargo, la Tecnología Educativa, como disciplina en la que se sustenta la EaD, no ha producido o generado aún un conocimiento suficientemente coherente y explicativo del conjunto de fenómenos culturales y educativos relacionados con estas nuevas prácticas, tal como lo expresa Area Moreira:

“Podemos afirmar que hasta la fecha el conocimiento que hemos producido sobre los medios y tecnologías en la educación es excesivamente fragmentado y ha sido de poca utilidad para generar procesos de mejora y cambio educativo" (Moreira, 2003, p. 1).

Por ello a la EaD, y por supuesto a quienes trabajen para su desarrollo, les cabe la responsabilidad de decidir si se va a optar por modelos que pongan el acento en la adquisición de información o en el desarrollo del pensamiento crítico capaz de usar la información para pensar y actuar comprometida y creativamente en la transformación social con sentido democrático, cuyos valores esenciales son la justicia y la libertad. Ello implica tam- 
bién decidir si se trabaja por y para la reproducción del orden social impuesto, a través de un modelo industrial de la EaD, o si se está dispuesto a buscar nuevos modelos, más abiertos que incorporen al estudiante como sujeto activo, deseante, productivo y al docente como mediador, crítico y creativo, entre el alumno y la cultura, organizada y distribuida en el currículum, como conocimiento para ser enseñando.

Se trata entonces de la construcción de una didáctica de la EaD que desde una perspectiva sociocrítica atienda a sus funciones éticas y políticas, al tiempo que "recupere la unidad perdida entre contenidos, metodologías y objetivos de la enseñanza" (Brovelli, 1990, p. 4). Esta construcción tendrá que atender tanto a los principios de igualdad y democracia ante la educación, a los derechos de los sujetos y a la justicia social, como a la aceptación de las diferencias, a la subjetividad en los procesos de aprendizaje, a la pluralidad cultural.

\section{Para ir pensando una nueva didáctica de la EaD en la educación superior}

Un buen ejercicio es comenzar con preguntas que orienten nuestra búsqueda y faciliten la creación de nuevas propuestas didácticas: ¿Cómo crear situaciones de enseñanza y de aprendizaje que promuevan el pensamiento crítico y creativo?, Cómo dialectizar las relaciones entre los tres polos de la tríada didáctica?, ¿Cómo atender a la lógica de la disciplina y crear una lógica de la interacción que lleven a un tipo de conocimiento procesual y crítico?, ¿Cómo pensar la lógica de la interacción mediada por la distancia y las nuevas tecnologías en situaciones de enseñanza y aprendizaje asincrónico?, ¿Cómo facilitar en el estudiante una relación de interioridad con el conocimiento?, ¿Cómo promover interrelaciones entre los aportes teóricos y la reflexión acerca de las prácticas profesionales en cursos de posgrado?, ¿Cómo atender a las dimensiones sociales, políticas y éticas de la enseñanza a través de las propuestas que se elaboren de las formas de evaluación que se utilicen?

Las preguntas pueden continuar, pero también es importante comenzar a ensayar algunas respuestas como las siguientes: 1) El estudiante tiene que ser percibido como sujeto activo, capaz de comprender significados, de producir textos, realizar reflexiones; 2) Habrá que tener en cuenta que el conocimiento puede construirse subjetiva y colectivamente, a partir de la intersubjetividad, con distintas miradas y desde diferentes puntos de vista; 3) El docente, como sujeto que media entre el conocimiento y el alumno tendrá que facilitar y orientar el estudio y la investigación, buscando la apertura conceptual, lo que requiere del trabajo con distintas fuentes e instrumentos; 4) La construcción metodológica que realice el docente dependerá fundamentalmente del dominio de los conocimientos que pretende enseñar y de 
la variedad de propuestas y estrategias de estudio, de los medios que utilice y de la coherencia y pertinencia entre ellos; 5) La propuesta de enseñanza y de aprendizaje tiene que atender a la contextualización de los conocimientos y a las relaciones entre la teoría y la práctica; 6) El sistema de comunicación tiene que ser fluido, buscando las relaciones en redes, lo que supone interrelaciones entre los docentes y los alumnos y de los alumnos entre sí; 7) Es importante tener presente que en la EaD se da un protagonismo constante de un conjunto de mediaciones pedagógicas e interacciones entre docentes, estudiantes, materiales, contenidos y medios; 8) El trabajo en EaD exige, como toda tarea social, la autenticidad de los actos de sus participantes y el someter a juicio crítico las argumentaciones que se produzcan.

\section{Desafíos didácticos que se le presentan a los profesores ante la EaD}

Son dos los desafíos didácticos que se le presentan a los profesores ante la educación a distancia. En primer lugar, tiene que preguntarse: ¿Quiénes son estos estudiantes que no tengo aquí y ahora en mi presencia material y directa?; y en segundo lugar, ¿Cómo construir propuestas didácticas que favorezcan el aprendizaje autónomo y el pensamiento crítico?

En un principio, el docente se ve ante una situación distinta a la que pueden describir en la mayoría de los discursos teóricos y prácticos acerca de la enseñanza. Tiene que establecer modos de comunicación diferentes a los que se pueden dar en el contexto de la clase tal como la conocemos en el sistema de enseñanza presencial. En el caso de la EaD se trata de una comunicación 'asincrónica' en cuanto a la categoría de tiempo, y 'sin territorialización' en cuanto al espacio. Esto que implica un cambio en lo referente al concepto de 'contexto de aprendizaje'. Sin embargo, sabe que la comunicación que se logre tendrá que ser fluida y abierta, de modo que facilite y propicie la participación activa del estudiante en las propuestas de enseñanza que formule. Tiene que poder imaginar estudiantes diferentes, con lo que también variarán las formas de relacionarse con ellos y, por lo tanto, también serán distintas las respuestas que pueda recibir.

Para comenzar desde el lugar de la posibilidad de la enseñanza y el aprendizaje, es pertinente señalar que el estudiante que opta por un sistema a distancia es una persona que ha tomado una decisión autónoma. Asimismo, está dispuesto a iniciar un camino de aprendizaje y estudio que será diferente al que seguramente está acostumbrado. Por ello, está dispuesto a intentar otras formas de trabajo. O sea, contamos con 'un sujeto que desea aprender' de otra manera y está dispuesto a recibir propuestas de enseñanza y de aprendizaje que requerirán de otras formas de trabajo, que deberán compatibilizarse con las ya adquiridas, poniendo en juego todo su potencial cognos- 
citivo. El sujeto de aprendizaje será el responsable de manejar y armar su contexto para aprender, al que sólo de manera indirecta, el docente puede aportar y sugerir algunos elementos que pueden pasar a formar parte de él.

El estudiante en la EaD es, entonces, un sujeto adulto con motivación suficiente (interna y externa) para iniciar una determinada formación. Posee un capital cultural - desconocido para el docente, pero que tendrá que poder indagar por diferentes medios. Éste último actuará como sustento y base de los nuevos aprendizajes, ya que cuenta con representaciones y significados construidos anteriormente. Éstos podrán ponerse en juego ante las nuevas propuestas de aprendizaje y, seguramente, ya habrán configurado estilos de aprendizaje sobre los que van a actuar los nuevos requerimientos del sistema a distancia, modificándolos, ampliándolos, haciéndolos más complejos, pero nunca negándolos.

Retomando planteos anteriores, diremos que el estudiante para aprender deberá enfrentarse con una 'lógica de los contenidos', cuya presentación y desarrollo tendrá que ver con la propuesta de enseñanza que haya elaborado el docente y con una 'lógica de la interacción' con los contenidos y con las personas, que en gran parte también depende de la propuesta del docente y de la misma modalidad de EaD por la que se haya optado. La interacción que se provoque dependerá de cuan abierta o cerrada sea la modalidad de enseñanza y aprendizaje presentada en relación con los objetos de conocimiento.

De todos modos, es importante reconocer que en la EaD se produce, inevitablemente, un proceso de individuación en el aprendizaje, o mejor dicho, en el aprendizaje autodirigido, ya que es el estudiante la oportunidad de aprender, el ritmo y las condiciones en las que se produce el aprendizaje. También habrá que considerar la posibilidad del surgimiento de problemas, obstáculos o conflictos que puede tener el estudiante frente a la propuesta de aprendizaje. De allí que sea necesario pensar en la posibilidad de que ante estas situaciones el estudiante pueda interactuar con los docentes (incluidos los tutores), como mediadores capaces de ofrecer las orientaciones adecuadas y oportunas para que el proceso de aprendizaje tenga la continuidad necesaria.

Cabe subrayar dos premisas importantes a tener en cuenta, si es que se pretende lograr un proceso de aprendizaje significativo y valioso: 1) tener presente que los estudiantes son sujetos activos, con conocimientos previos, capaces de confrontar saberes y de construir y resignificar nuevos conocimientos; y 2) propiciar actividades de aprendizaje grupal mediante foros, chats o encuentros presenciales, que eviten o atenúen la situación de aislamiento o soledad que puede producir la distancia social, temporal y espacial.

Otro aspecto al que se hace referencia es cómo construir propuestas didácticas que favorezcan el aprendizaje autónomo y el pensamiento crítico. 
Un primer problema que se le presenta al docente en la EaD es articular contenido y formas de presentarlo y trabajarlo de manera coherente y pertinente. Al docente le corresponderá trabajar fundamentalmente con la 'significatividad lógica' del contenido a ser aprendido — de ahí la importancia de su selección y organización, suponiendo que el estudiante ya motivado podrá otorgarle la 'significatividad psicológica' (Ausubel, 1982, p. 63), en tanto ambas son necesarias para logro de aprendizajes deseados. Para ello habrá que buscar formas de presentación de los contenidos y de las actividades para realizar con ellos, que atiendan tanto a la adquisición de conceptos, como a la de procedimientos adecuados y al desarrollo de actitudes y valores que ubiquen al sujeto de aprendizaje como sujeto social, comprometido con su entorno y con su tiempo.

Si bien en la EaD se mantiene como estructura básica la tríada didáctica (docente-alumno-conocimiento), la tarea mediadora del docente requiere de un cambio en la manera de concebirla y en los modos de realizarla. Las mediaciones pedagógicas están constituidas por el conjunto de acciones, interacciones, recursos y materiales didácticos, que se presentan como un conjunto articulado de componentes que intervienen en el proceso de enseñanza y de aprendizaje (Fainholc, 1999, p. 49). De modo que en la EaD el docente, en primer lugar, tiene que pensar en otro tipo de recursos y medios tecnológicos que ayuden a hacer más clara, efectiva y pertinente la mediación, debiendo soportar la incertidumbre de no tener una respuesta inmediata, ya que aquí puede estar suspendida en el tiempo y en un espacio simbólico en el que está ausente la relación cara a cara entre docente y alumno. El texto de enseñanza (ya sea online o impreso) adquiere una importancia relevante en tanto es el soporte fundamental y no se tiene la oportunidad de cambiarlo, retraducirlo o enriquecerlo en el momento en que se produce el encuentro entre el sujeto de aprendizaje y el texto. Así, tendrán lugar nuevos modos de percepción y lenguaje, nuevas narrativas y escrituras.

Lo anteriormente expresado muestra la importancia que cobra, especialmente en la EaD, el 'diseño de la enseñanza y del aprendizaje'. Si por diseño se entiende la capacidad de imaginar, prever, anticipar, quiere decir que habrá que poner en juego en esta actividad todos los conocimientos que el docente disponga: aquellos referidos a los procesos de aprendizaje de los estudiantes, los relacionados con el contenido a ser enseñado, los que tengan que ver con los modos de conocimiento y con las formas de presentarlo, con la creatividad en las propuestas de actividades y de evaluación. La integración y la coherencia entre todos estos aspectos serán fundamentales para la elaboración de diseños de enseñanza y de aprendizaje, tendientes al logro de buena calidad en los procesos y resultados del aprendizaje.

Para ello el docente tiene que ofrecer formas de andamiaje del conocimiento, de modo de prestar la ayuda necesaria para el aprendizaje, tenien- 
do claro cuando retirarla, porque se supone que el alumno ya puede hacer solo lo que se le propone. Al mismo tiempo, el docente con su propuesta de enseñanza deberá pretender que el sujeto de aprendizaje, partiendo de los conocimientos y habilidades de que dispone, trabaje de modo tal que alcance el máximo de sus posibilidades de aprendizaje reflexivo, complejo y crítico. Es decir, que alcance la 'zona de desarrollo próximo' en palabras de Vigotsky.

Otro aspecto a tener en cuenta en la tarea de diseño es referido al planteo de estrategias 'metacognitivas', que le permitan al estudiante realizar, periódicamente, actividades de meta análisis, tanto acerca de los conocimientos aprendidos, como de los procesos a través de los cuales considera que aprendió. Esto es lo que permite realmente saber si se ha aprendido, de modo que se convierte en la mejor situación de autoevaluación, condición necesaria para seguir aprendiendo en mejores condiciones. De este modo, el docente puede desplazarse del lugar del 'control del aprendizaje', para ubicarse en el de 'orientador de los procesos' realizados por los estudiantes de acuerdo con sus necesidades.

En el caso de la educación superior, es una condición necesaria el trabajo de investigación de fuentes y de campo o de laboratorio, según el tipo de conocimiento de que se trate. El texto de enseñanza tiene función de ubicar al estudiante ante el conocimiento y la propuesta de trabajo que se realice, al tiempo que orientarlo en el proceso de aprendizaje y de investigación, y de ayudarlo en el planteo y solución de problemas, en la elaboración de informes, ensayos, proyectos, etc. Por lo expuesto es que en la EaD, al menos en el caso de la educación superior, los materiales de enseñanza y de aprendizaje no deben ser presentados como un producto envasado y cerrado, sino que debe ser una invitación al estudio y la indagación de diferentes fuentes que induzcan al pensamiento reflexivo y crítico, a la formulación de nuevos interrogantes, que abran el camino a procesos investigativos.

\section{Consideraciones finales}

Es necesario, finalmente, hacer referencia al carácter complejo del conocimiento, a su multidimensionalidad y a la carga de incertidumbre que muy posiblemente se produzca como consecuencia de estas notas propias del conocimiento. De ahí que no se pueda presentar los conocimientos como cerrados y empaquetados, sino que la propuesta de estudio tendrá que aceptar e incentivar el riesgo, el azar, la iniciativa, la decisión, las críticas y las autocríticas... Tal como lo expresa Morin (2001, p. 17): “Es necesario aprender a navegar en un océano de incertidumbres a través de archipiélagos de certezas". 
La enseñanza y el desarrollo de la capacidad para argumentar y contraargumentar, para el análisis de las contradicciones, el análisis del discurso de los otros y la reflexión acerca del propio, se convierten en condiciones necesarias de la enseñanza, sea ésta presencial o a distancia. A ello debemos agregar la formación en valores que hagan a la búsqueda constante de mayor y más auténtica democracia, a la convivencia social en paz y al accionar profesional comprometido y crítico.

Lo que intento buscar, e invito a seguir trabajando en ello, es en un 'modelo' pedagógico que más que poner el acento en el docente o en el alumno, se centre en la relación entre ambos, es decir, en los distintos modos de interrelación de los actores pedagógicos, que rompan con la directividad e intenten dar lugar a situaciones de subjetivación. Esto es lo que nos permitiría pensar en verdaderos procesos formativos.

\section{Nota}

1 Investigadora y docente de la Facultad de Humanidades y Artes de la Universidad Nacional del Rosario, Argentina. Doctora en Ciencias de la Educación en la Universidad Nacional de Educación a Distancia, España. <mbrovell@arnet.com.ar> 


\section{Referências}

APARICI, Roberto. 2002. Mitos de la educación a distancia y de las nuevas tecnologías. Revista Iberoamericana de Educación a Distancia (Ried), v. 5, n. 1.

AUSUBEL, David P. 1982. Psicología educativa. Madrid: Trillas.

BARRIGA, Ángel D. 1986. Didáctica y currículum. México: Nuevo Mar.

BEILLEROT, Jacky. 1996. La formación de formadores. Buenos Aires: Ediciones Novedades Educativas/Facultad de Filosofía y Letras de la Universidad de Buenos Aires.

BROVELLI, Marta. 1990. Aportes acerca del problema de la didáctica. Cuaderno de Formación Docente, n. 11, p. 1-19.

BRUNER, Jerome. 1990. Actos de significado. Más allá de la revolución cognitiva. Madrid: Alianza.

CARR, Wilfred; KEMMIS, Stephen. 1988. Teoría crítica de la enseñanza. Barcelona: Martínez Roca.

CASTELLS, Manuel. 1994. Flujos, redes e identidades: una teoría crítica de la sociedad informacional. In: CASTELLS, Manuel (org.). Nuevas perspectivas criticas en educación. Barcelona: Paidós (Educador).

CULLEN, Carlos. 1997. Crítica de las razones de educar. Buenos Aires: Paidós (Cuestiones de Educación).
CHEVALARD, Yves. 1997 La transposición didáctica. Buenos Aires: Aique.

EDWARDS, Derek; MERCER, Neil. 1988. El conocimiento compartido. Barcelona: Paidós/MEC (Temas de Educación).

FAINHOLC, Beatriz. 1999. La interactividad en la educación a distancia. Buenos Aires: Paidós (Cuestiones de Educación).

GASKINS, Irene; ELLIOT, Thorne. 1999. Cómo enseñar estrategias metacognitivas en la escuela. Buenos Aires: Paidós (Cuestiones de Educación).

LITWIN, Edith (org.). 1995. Tecnología educativa: política, historias, propuestas. Buenos Aires: Paidós (Cuestiones de Educación).

MOREIRA, Manuel A. 2003. Tecnología educativa y posmodernidad. Buscando nuevas bases conceptuales en tiempos de cambio. Universidad de la Laguna, Islas Canarias. Disponible en: $<$ webpage.ull.es/user/manarea $>$. Acceso en: 2 ene. 2005.

MORIN, Edgard. 2001. Los siete saberes necesarios para la educación del futuro. Buenos Aires: Nueva Visión.

VIGOTSKY, Lev S. 1979. Los procesos psicológicos superiores. Barcelona: Salvá. 